\title{
Experimental and numerical study of circular, stainless thin tube energy absorber under axial impact by a control rod
}

\author{
Zhe Yang ${ }^{\mathrm{a}}$, He Yan ${ }^{\mathrm{b}}$, Chenguang Huang ${ }^{\mathrm{a}}$, Xingzhong Diao ${ }^{\mathrm{b}}$, Xianqian $\mathrm{Wu}^{\mathrm{a}}$, \\ Shaohua Wang ${ }^{\mathrm{b}}$, Lingling $\mathrm{Lu}^{\mathrm{a}}$, Lijuan Liao ${ }^{\mathrm{a}}$, Yanpeng Wei ${ }^{\mathrm{a}, *}$ \\ a Key Laboratory for Mechanics in Fluid Solid Coupling Systems, Institute of Mechanics, Chinese Academy of Sciences, Beijing 100190, China \\ ${ }^{\mathrm{b}}$ Institute of Nuclear and New Energy Technology of Tsinghua University, Key Laboratory of Advanced Reactor Engineering and Safety, Ministry of Education, \\ Beijing 100084, China
}

\section{A R T I C L E I N F O}

\section{Article history:}

Received 27 December 2013

Received in revised form

27 March 2014

Accepted 27 March 2014

Available online 25 April 2014

Keywords:

Circular thin tube

Energy absorber

Progressive buckling

Axial impact

Strain rate

Interfacial pressure

\begin{abstract}
A B S T R A C T
In this study, several crashworthiness parameters of a circular, thin tube energy-absorbing structure, which is used in a high-temperature, gas-cooled reactor (HTR), are studied experimentally and numerically at various tube thicknesses, temperatures and impact velocities. The average crushing force is fundamentally dependent on strain hardening, strain rate hardening, and, particularly, temperature softening of the material. The peak forces during buckling are significantly affected by the local strain rate in the material and exhibit a decreasing trend in sequentially formed folds. Reducing the tube thickness is an effective method to weaken the average crushing force, but it does not weaken the maximum crushing force. Additionally, the stress concentration at the edge of the backplate-graphite contact surface is evaluated in detail to ensure the structural security of the energy absorber.
\end{abstract}

(c) 2014 Elsevier Ltd. All rights reserved.

\section{Introduction}

Impact is a critical issue in the design of control systems in nuclear power plants $[1,2]$. The control rods, which are slender and massive, are inserted into the reactor via a free fall once a shutdown signal is triggered. The free-fall control rod, after travelling through the nuclear reaction zone, will substantially impact the graphite reactor structure $[3,4]$. This phenomenon is more serious in a high-temperature gas-cooled reactor (HTR) [5,6] because there is no liquid to decelerate the control rod in the nuclear reaction zone. Therefore, an impact energy absorber is required between the control rod and the graphite reactor to weaken the impact force and disperse the impact energy. Furthermore, due to the low strength and brittleness of graphite, which is the main constituent material of the reactor, and the ultra-clean spaces required for a nuclear reaction, the design demands the following:

i. The pressure imposed on the reactor structure during the entire impact event is strictly limited below the maximum allowable stress of graphite at all locations.

\footnotetext{
* Corresponding author. Tel.: +861082544256.

E-mail addresses: Huangcg@imech.ac.cn (C. Huang), weiyanpeng@imech.ac.cn (Y. Wei).
}

ii. The absorber structure must exhibit good stability under extremely high environmental temperatures.

iii. There must be no debris or other additional products during impact energy absorption.

Circular thin tubes, which are the most efficient and reliable energy absorbers, may be an appropriate structure to service in such strict conditions [7-9]. From the perspective of an energy absorption capacity, it has been found that a circular, thin tube under axial compression is one of the best devices because it provides a reasonably high specific energy $\left(\sim 3 \times 10^{4} \mathrm{Nm} / \mathrm{kg}\right)$, high volumetric efficiency (0.7-0.8) and has a simple geometry [10]. More importantly, its progressive buckling behavior can provide approximately a constant crushing force, which is the prime characteristic of the energy absorber for our purposes. At quasistatic conditions, due to the complexity of plastic hinge formation mechanism, the evaluated average crushing force is different for different buckling modes (Concertina, diamond and mixed). Alexander presented a rigid-plastic analysis to theoretically estimate the concertina mode [11], which is the most famous equation for energy absorption criteria in axial crushing of circular tubes. Abramowicz and Jones [12] improved Alexander's analysis, which gave a more consistent agreement with experimental results. Pugsley and Macaulay [13] presented an expression for the 


\begin{tabular}{|c|c|c|c|}
\hline \multicolumn{2}{|c|}{ Nomenclature } & $\varepsilon$ & equivalent plastic strain \\
\hline$D$ & diameter of tube & $\dot{\varepsilon}_{0}$ & reference strain rate, $1 \mathrm{~s}^{-1}$ \\
\hline$D_{1}$ & diameter of backplate & $\dot{\varepsilon}_{0}^{*}$ & dimensionless strain rate \\
\hline$h$ & thickness of tube & $\mu$ & Poisson's ratio \\
\hline$L$ & length of tube & $V$ & impact velocity \\
\hline$H$ & length of control rod & $E_{\text {in }}$ & input kinetic energy \\
\hline$\Phi$ & diameter of control rod & $T$ & environmental temperature \\
\hline$S$ & area of backplate & $T_{r}$ & room temperature \\
\hline$a$ & length of graphite & $T_{m}$ & melting temperature of stainless steel \\
\hline Y & yield strength of stainless steel & $T^{*}$ & homologous temperature \\
\hline$\rho$ & density of stainless steel & $F_{\max }$ & maximum transient crushing force \\
\hline$E$ & Young's modulus of stainless steel & $F_{a v}$ & average crushing force \\
\hline$E_{1}$ & Young's modulus of graphite & $S_{\max }$ & maximum crushing displacement \\
\hline$M$ & Mass of control rod & $P_{\max }$ & maximum interfacial pressure \\
\hline$m$ & Mass of backplate & $P_{a v}$ & average interfacial pressure \\
\hline$\sigma_{e q}$ & von Mises flow stress & & \\
\hline
\end{tabular}

average crushing force by assuming that kinetic energy is absorbed by plastic bending and shear of the diamond patterns.

These equations are also suitable a low-velocity impact, where kinetic energy is considered to be converted into plastic work at the quasi-static. In this case, the inertia effect of the structure can be ignored; however, the effect of the loading condition on the mechanical properties of the materials must be considered. Most of the previous work has studied the effect of the average strain rate on the increase in yield stress [14] using the Cowper-Symonds equation [15]. Nevertheless, little work has been done on the effect of temperature. Though, similar to the average crushing force, which is a decisive factor for energy absorption, the transient crushing force is equally important in the design of absorbers because it incurs the maximum load (often occurring at the initiation of the buckling process) that graphite bears. A numbers of works have studied several buckling features and crushing forces of thin tubes subjected to axial impact loads $[16,17]$, including studying the effect of impact velocity, shell geometry, etc. Generally, the profile of the transient crushing force can reflect the non-uniform deformation characteristics of the buckling process, including the initiation, development and termination of the plastic hinges. At an impact condition, these characteristics are manifested as a non-uniform strain rate in the materials. Its effect on the buckling process and crushing force profile has not been fully understood. Another vital factor is the contact pressure at the interface between the absorber and graphite. Due to the higher stiffness of stainless steel, the backplate of the absorber will slightly penetrate into the graphite block during the buckling process, which leads to a non-uniform stress distribution in the backplate-graphite contact region, which requires a detailed understanding of the time and spatial pressure distribution at the absorber-graphite interface.

In this paper, an energy-absorbing structure based on the progressive buckling phenomena of circular, thin tubes is explored to protect graphite from a low-speed impact from a massive control rod. Several crashworthiness parameters, including the average and transient crushing force and interfacial pressures, are studied experimentally and numerically for various impact velocities, temperatures and geometries. The effects of strain rate and temperature on crushing loads characteristics are discussed in detail in the study.

\section{Structure of the absorber}

As shown in Fig. 1, the energy absorber is composed of a buffer ring, circular, thin tube and a backplate. The inclined surface of the buffer ring is used to weaken the head pressure of the stress wave generated by impact. The backplate is used for the homogenization of the interface pressure between the absorber and graphite. To ensure stability, both ends of the thin tube are inserted into card slots in the buffer ring and backplate. An artificial defect ( $1 \mathrm{~mm}$ width dimple along the entire circumference) is prefabricated at the proximal end of the thin tube to control the start of buckling. The dimensions of the evaluated thin tubes are length $L=450 \mathrm{~mm}$ and diameter $D=80 \mathrm{~mm}$ and various thicknesses (as shown in Table 2).

All the components of the absorber, including the buffer ring, thin tube and backplate, were made of stainless steel with a density $\rho=7930 \mathrm{~kg} / \mathrm{m}^{3}$, Young's modulus $E=210 \mathrm{GPa}$ and Poisson's ratio $\mu=0.3$.

\section{Experiments}

The impact experiments were performed at set conditions ( $h=2 \mathrm{~mm}, V=13 \mathrm{~m} / \mathrm{s}$ and $T=300 \mathrm{~K}$ ). The impact load was generated by a modified control rod, which was made of low-carbon steel with a total length $H=3000 \mathrm{~mm}$, diameter $\Phi=110 \mathrm{~mm}$ and mass $M=200 \mathrm{~kg}$. The head of the rod was also machined to an inclined surface to fit the buffer ring. As shown in Fig. 2, the

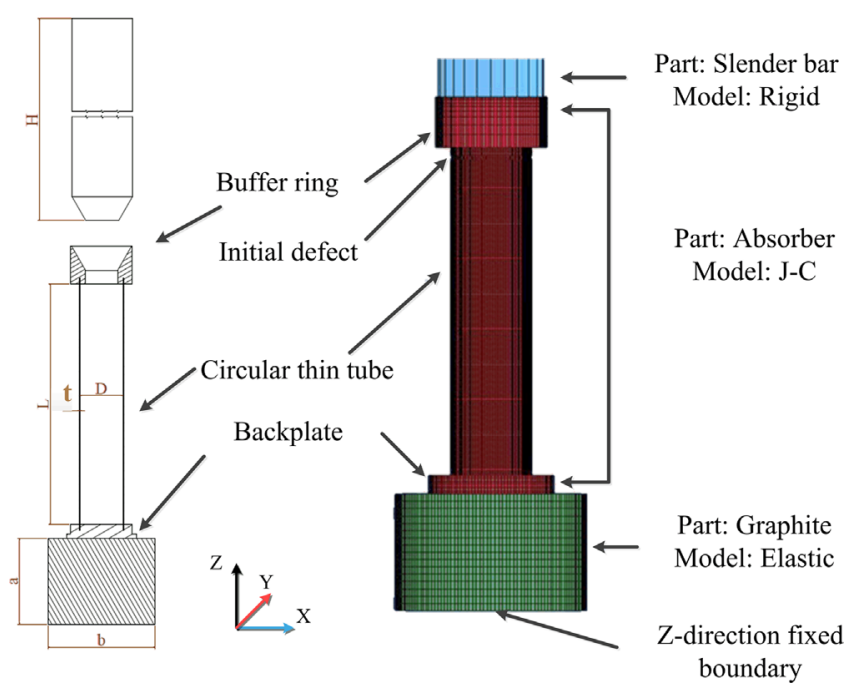

Fig. 1. Schematic structure of the energy absorber and numerical model. 


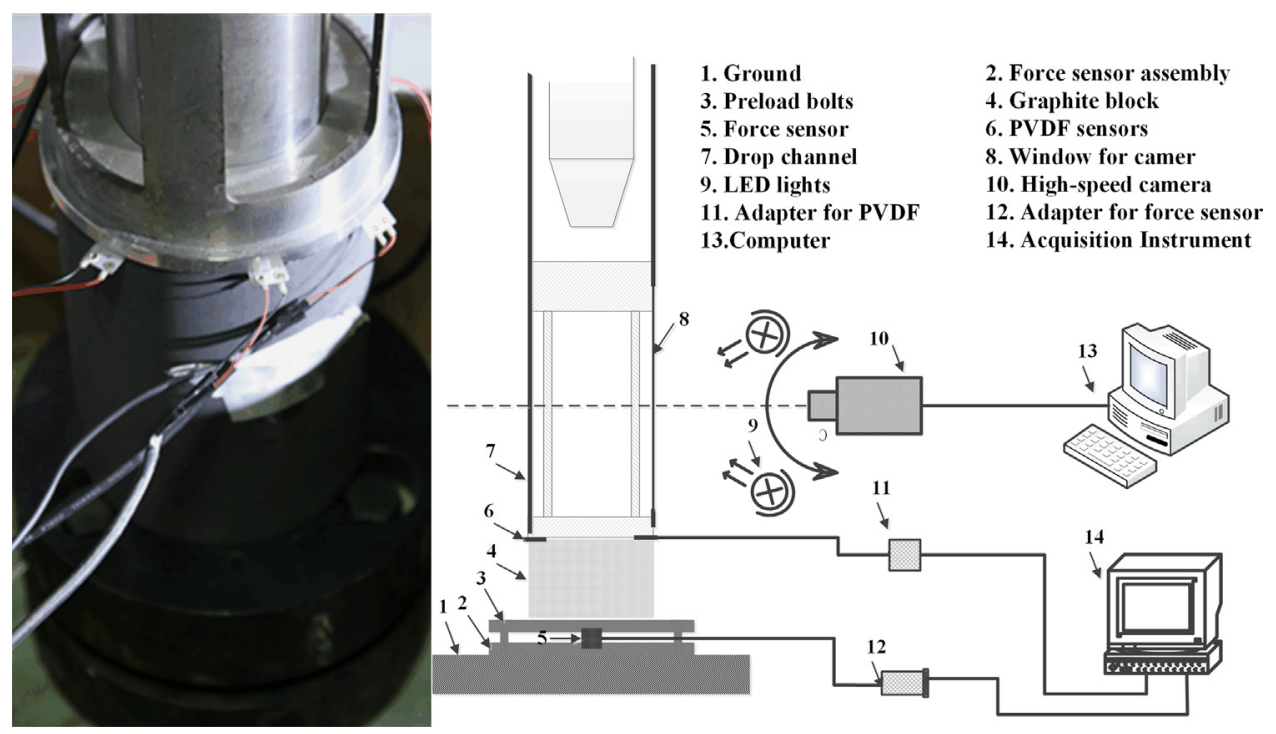

Fig. 2. Schematic diagram of the experimental setup.

control rod is dropped from a height of $8.7 \mathrm{~m}$ via the acceleration of gravity, reaching a velocity $V \approx 13 \mathrm{~m} / \mathrm{s}$ before impact. To prevent the control rod and energy absorber from laterally capsizing after impact, both were contained within a drop channel with a $12,000-$ $\mathrm{mm}$ length and $120-\mathrm{mm}$ diameter. The graphite block, with a 200$\mathrm{mm}$ diameter and $160-\mathrm{mm}$ length, was located between the absorber and reinforced-concrete floor. Multiple test methods were performed to capture the dynamic response of the structure.

\subsection{High-speed imaging of buckling process}

The progressive buckling process was captured by a high-speed camera with $5700 \mathrm{fps}$ at full resolution $(1024 \times 1024$ pixel). A mode of $15,000 \mathrm{fps}$ at a resolution of $1024 \times 258$ pixel was selected to capture the detailed deformation characteristics of the buckling behavior through a window on the drop channel. Two $200 \mathrm{~W}$ white LEDs (19,000 lm for each LED) were used as light sources to ensure an adequate luminous flux was supplied to CCD for such high speeds.

\subsection{Transient crushing force measurement}

A pressure sensor assembly is located at the bottom of the graphite block to measure the transient crushing force during the buckling process. The pressure sensor is made of manganin with a piezoelectric coefficient of $3000 \mathrm{~N} / \mathrm{V}$. As shown in Fig. 2, the sensor is sandwiched by two steel plates and preloaded with $3000 \mathrm{~N}$ (which will be subtracted from the test results by resetting the baseline in the data acquisition software) by six bolts to ensure the stability of the assembly.

All the voltage signals were obtain by an NI acquisition instrument with a $1 \mathrm{MHz}$ sampling frequency and 12 bit sampling accuracy.

\subsection{Transient interfacial pressure measurements}

Six polymer polyvinylidenefluoride (PVDF) pressure gauges are used to capture the transient pressure at the boundary of the backplate-graphite. The construction of the PVDF gauges is shown in Fig. 3. A $25 \mu \mathrm{m}$ thick aluminum-metalized polarized PVDF film is encapsulated by two layers of Mylar film. The gauge legs are leads from the upper and lower aluminum-metalized surface. The total thickness of the gauge package is approximately $0.1 \mu \mathrm{m}$.

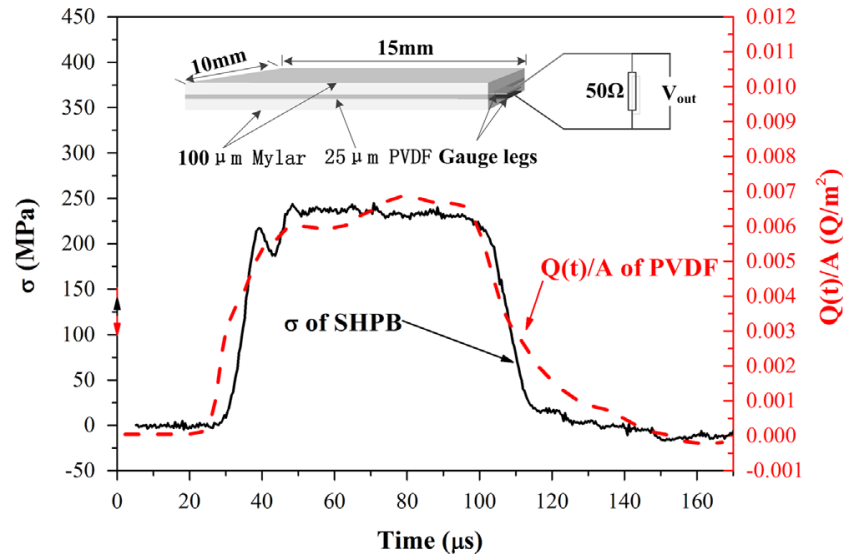

Fig. 3. Construction and calibration curve of the PVDF gauges.

A large enough sensitive area $(10 \mathrm{~mm} \times 15 \mathrm{~mm})$ was selected to minimize the loading effect [18]. Additionally, a simple matching circuit is used to transfer the current into voltage signals, which can be easily acquired by an oscilloscope. However, the response frequency and the piezoelectric coefficient of the PVDF, which is closely related to the thickness and sound speed of the PVDF, assembly process of the gauge and loading condition, is required to calibrate before running an experiment. Dynamic calibration of the PVDF gauge was accomplished by the collision of an elastic steel bar using a Hopkinson bar. The elastic stress wave signal from the strain gauge had a rising time of approximately $10 \mu \mathrm{s}$, average pressure of approximately $220 \mathrm{MPa}$ and duration time of approximately $80 \mu \mathrm{m}$. As shown in Fig. 3, the PVDF gauge exhibits good linearity (calibrated piezoelectric coefficient is $24 \mathrm{pC} / \mathrm{N}$ ), below $100 \mathrm{kHz}$.

\section{Finite element simulation}

The dynamic simulations were performed using the FE code, ANSYS/LS-DYNA 3D. As shown in Fig. 1, three parts are defined, including the control rod, energy absorber and graphite block. Eight-node hexahedral elements were selected for all the parts. The meshes are relatively sparse for the control rod, whose deformation is not our focus. All thin tubes are modeled with 
Table 1

Materials parameters for each part in the numerical model.

\begin{tabular}{llllll}
\hline Parts & Material & Constitutive model & $\boldsymbol{E}(\mathbf{G P a})$ & $\boldsymbol{\rho}\left(\mathbf{k g} / \mathbf{m}^{\mathbf{3}}\right)$ & $\boldsymbol{\mu}$ \\
\hline Control rod & Low-carbon steel & Rigid & 210 & 7830 & 0.3 \\
Absorber & 304 stainless steel & Johnson-Cook & 210 & 7930 & 0.3 \\
Graphite & Graphite & Elastic & 9.04 & $1760[19]$ & 0.2 \\
\hline
\end{tabular}

Table 2

Cases assessed with a numerical simulation in the present study.

\begin{tabular}{lllll}
\hline No. & $\begin{array}{l}\text { Shell thickness } \\
h(\mathbf{m m})\end{array}$ & $\begin{array}{l}\text { Initial velocity } \\
\boldsymbol{V}(\mathbf{m} / \mathbf{s})\end{array}$ & $\begin{array}{l}\text { Average strain } \\
\text { rate } \dot{\varepsilon}^{\mathrm{a}}\left(\mathbf{s}^{-\mathbf{1}}\right)\end{array}$ & $\begin{array}{l}\text { Temperature } \\
\boldsymbol{T}(\mathbf{K})\end{array}$ \\
\hline $\mathbf{1}$ & 1.2 & 13 & 120 & 300 \\
$\mathbf{2}$ & 1.5 & 13 & 120 & 300 \\
$\mathbf{3}^{\mathbf{b}}$ & 2.0 & 13 & 120 & 300 \\
$\mathbf{4}$ & 2.5 & 13 & 120 & 300 \\
$\mathbf{5}$ & 2.0 & 8 & 75 & 300 \\
$\mathbf{6}$ & 2.0 & 5 & 46 & 300 \\
$\mathbf{7}$ & 2.0 & 13 & 120 & 523 \\
$\mathbf{8}$ & 2.0 & 13 & 120 & 773 \\
\hline
\end{tabular}

a Calculated by $\dot{\varepsilon}=0.74 \mathrm{~V} / \mathrm{D}$ from reference [12].

b No. 3 was also experimentally evaluated.

200 elements in the longitudinal direction and four elements through the shell thickness. The contact (absorber-control rod and absorber-graphite) is defined using the "surface interaction" concept. Any self-contact at the inner and outer shell surfaces is assumed frictionless. The bottom surface of graphite is fixed in the $z$-direction to model a rigid boundary. The characteristics of the material for each part are listed in Table 1.

The von Mises flow stress of the material is described using the Johnson-Cook [20] constitutive model, which takes strain rate and temperature into account, and $\sigma_{e q}$ is expressed as

$\sigma_{e q}=\left(A+B \varepsilon^{n}\right)\left(1+C \ln \dot{\varepsilon}^{*}\right)\left[1-\left(T^{*}\right)^{m}\right]$

where $\varepsilon$ is the equivalent plastic strain, $\dot{\varepsilon}^{*}=\dot{\varepsilon} / \dot{\varepsilon}_{0}$ is the dimensionless plastic strain rate for $\dot{\varepsilon}_{0}=1 / \mathrm{s}, T^{*}=\left(T-T_{r}\right) /\left(T_{m}-T_{r}\right)$ is the homologous temperature for $T_{m}=1713 \mathrm{~K}$ and $T_{r}=293 \mathrm{~K}$. The expression in the first set of brackets gives the stress as a function of strain for $\dot{\varepsilon}^{*}=1$ and $T^{*}=0$. Expressions in the second and third sets of brackets represent the effects of strain rate and temperature, respectively. The data for the five material constants, $A, B, n, C$ and $m$, are obtained from static tensile tests and Hopkinson bar tests at elevated temperatures from reference [21].

All the cases evaluated in the numerical simulation are shown in Table 2.

\section{Results and discussions}

\subsection{Validity of numerical model}

Prototype verification of the impact event of the absorber by the control rod is complex and can be done systematically; however, there is no need to evaluate all the cases experimentally if an exact numerical model exists. In this section, the validity of the numerical model is assessed by experimental results at $h=2 \mathrm{~mm}, V=13 \mathrm{~m} / \mathrm{s}$ and $T=300 \mathrm{~K}$.

\subsubsection{Buckling modes}

When bearing an axial impact, a perfect thin tube will buckle from the distal end first, where the maximum load occurs due to the reflection of the stress wave [16], which significantly increases the chances of overturning, particularly for a slender control rod impact. Thus, prefabricated defects will weaken the local strength of the thin tube, which is an effective way to control the buckling process $[22,23]$. Both the experimental and numerical results show that the activation of the buckling process is well restricted to the proximal end, where the artificial dimple is located.

The diamond buckling mode is predicted well by the numerical models (Fig. 4(a)) at $h=2 \mathrm{~mm}, V=13 \mathrm{~m} / \mathrm{s}$ and $T=300 \mathrm{~K}$. Generally, thicker tubes (small $D / h$ ratio) tend to buckle in the concertina (axisymmetric) mode of deformation, whereas thinner tubes (high $D / h$ ratio) tend to buckle in diamond mode [9]. Several researchers have described experimental observations [24] and theoretical predictions [25] in response to the transition point from the concertina mode to diamond mode for intact, thin tubes. However, thus far, no exact analysis has been given to explain why a particular mode of deformation is adopted for a given tube with specific defects. The experimental results showed that the folds preferred diamond mode, which is predicted well by the numerical model, as shown in Fig. 4(a). Furthermore, this model can also capture the details of the plastic hinges for each fold (Fig. 4(b)-(d)).

\subsubsection{Crushing displacement}

With the help of a high-speed camera, the entire buckling process can be visually observed. The speed of the proximal end can be obtained by a two-dimensional digital image correlation (2D DIC) method. A speckled pattern was used to extract displacement information and is just the natural texture on the surface, i.e., no artificial techniques were used. However, a 2D DIC can only capture in-plane displacement on the planar surface. To meet the requirement, the tracking area is localized into a narrow band on the buffer ring, as shown in Fig. 5, which can be considered as a plane parallel to the CCD sensor during buckling. The buffer ring thickness $(70 \mathrm{~mm})$ is used as the calibration metric to determine the actual distance per pixel represented in the images.

Fig. 6 shows the typical displacement-time and velocity-time curve of the 2D DIC and numerical results. The motion trajectory of the proximal end can be divided into two stages: the progressive buckling stage and the rebound stage. In the first stage, the proximal end and control rod accelerate at a nearly constant value, $a \approx 620 \mathrm{~m} / \mathrm{s}^{2}$, until the crushing displacement reaches a maximum, $S_{\max }$. The numerical $S_{\max }$ is slightly larger than the experimental values, which is primarily due to the deviation in the initial impact speed in the experiments $(12.8 \mathrm{~m} / \mathrm{s}$ and the corresponding numerical condition was $13 \mathrm{~m} / \mathrm{s}$ ). All kinetic energy of the control rod is transferred into deformation energy of the thin tube until the proximal speed drops to $0 \mathrm{~m} / \mathrm{s}$. Afterwards, the elastic portion of the energy stored in both the buckled and unbuckled thin tube will release in the rebound stage during which the control rod will bounce back up and then drop for a second time. The elastic portion of energy, which is a small proportion of the total impact energy, will disperse by friction, and further deformation of the folds will occur. Due to the impact force in the bounce-back stage being relatively small (equivalent to the gravity of the control rod) compared with the progressive buckling stage, the calculation was performed at the moment very soon after $V=0 \mathrm{~m} / \mathrm{s}$. As shown in Fig. 6, there is good agreement of the crushing displacement and velocity during the progressive buckling process between the experimental and numerical results.

\subsubsection{Average crushing force}

The average crushing force is an important factor to evaluate the energy-absorbing capacity per unit length and can be expressed as

$F_{a v}=E_{i n} / S_{\max }$

where $E_{\text {in }}$ is the kinetic energy of the control rod. 

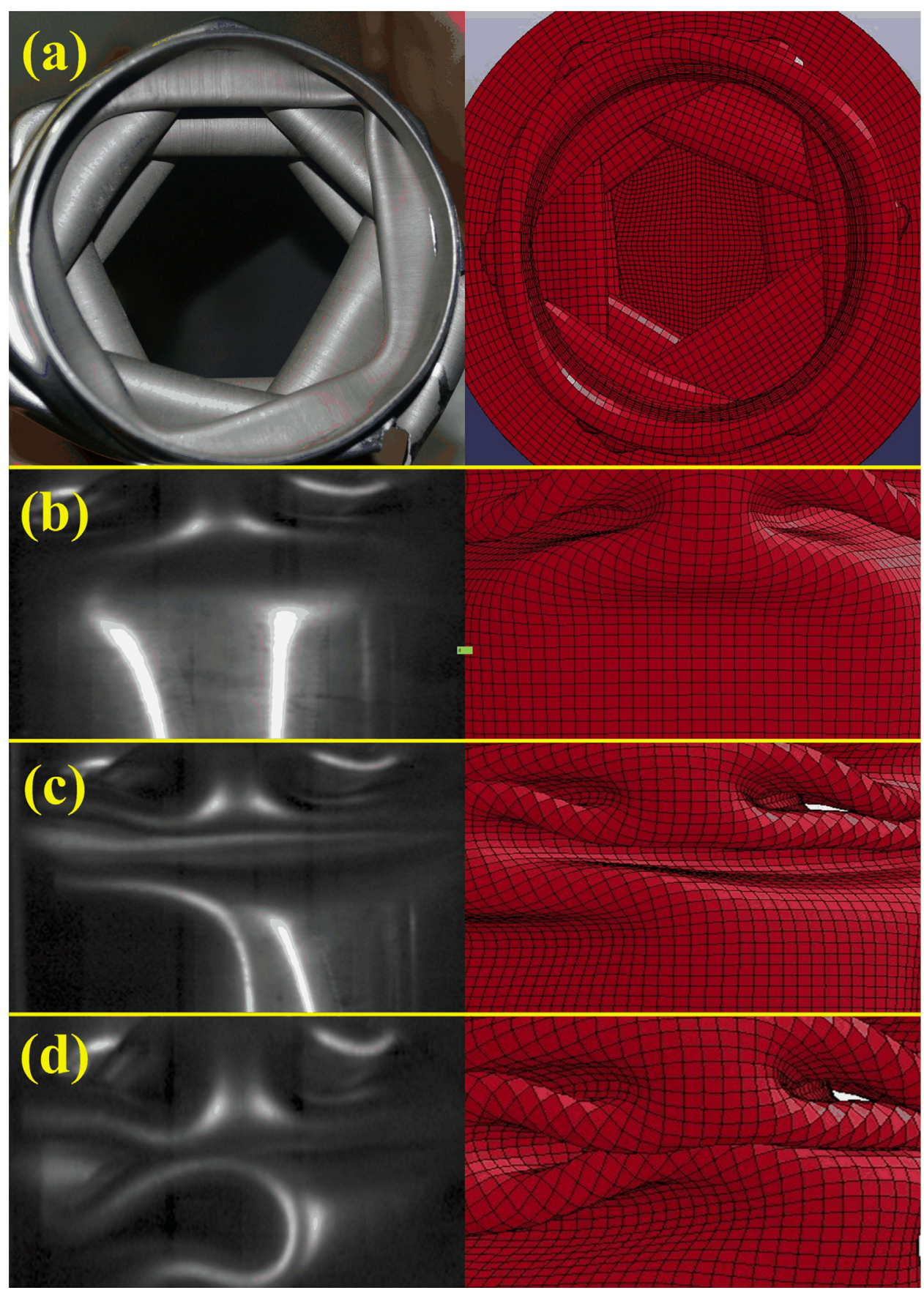

Fig. 4. Comparison of buckling modes and shapes of the plastic hinge. (a) Buckling modes, (b) 1st fold, (c) 2nd fold, (d) 3rd fold.

The numerical results are consistent with the experimental results but much higher than the theoretical prediction for the static diamond mode case by Wierzbicki [26], which obtained good agreement with experimental results. It is generally believed that average crushing force can be predicted by static theory in the case of a low-velocity impact. However, the effect of the loading conditions on the material properties must be included using a simple factor $\alpha$ [14]. This effect may include average strain rate hardening, strain hardening and temperature softening. Then we can get the average crushing force in low velocity impact and diamond mode case.

$$
F_{a v}=18.15 \alpha Y \delta^{2}(D / \delta)^{1 / 3}
$$

As shown in Fig. 7, the numerical values of $F_{a v}$ are plotted as thicknesses to compare with theoretical results. When $\alpha$ is equal to 1.779 , the results are extremely consistent at $V=13 \mathrm{~m} / \mathrm{s}$ and $T=300 \mathrm{~K}$, which demonstrates that if ignoring the inertial effect, $\alpha$ should be the only material parameter used to describe the effect of the loading condition on yield strength that is independent of the geometry of thin tubes.

Thus, $\alpha$ is not only derived from the average strain rate hardening effect during the buckling process but also from the hardening effect of the large strain during fold formation and the weakened effect of the environmental temperature. As shown in Table 3, $F_{a v}$ clearly decreases at higher environmental temperatures, which is primarily due to the yield strength and plastic flow stress decreasing as the temperature increases, which is a widely accepted softening effect of stainless steel. Although an increasing yield stress at elevated average strain rates is frequently reported for a large range of impact speeds, this strain rate hardening effect 


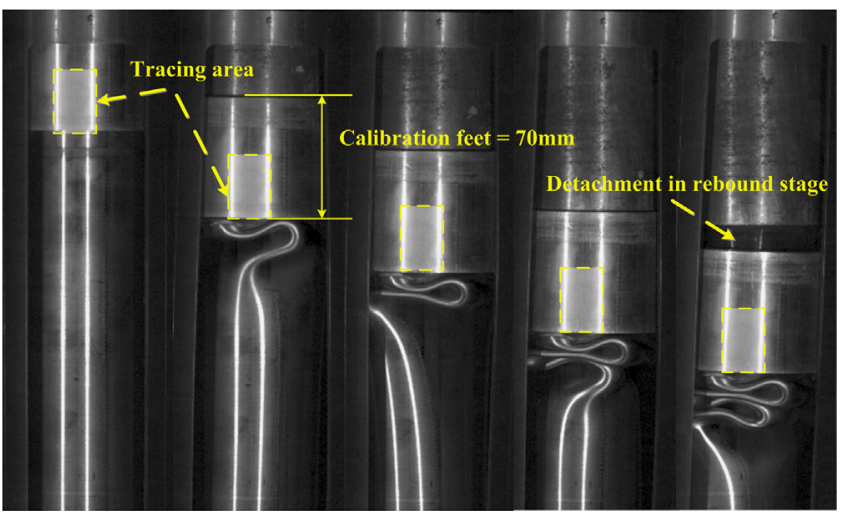

Fig. 5. Images of the buckling process captured by the high-speed camera.

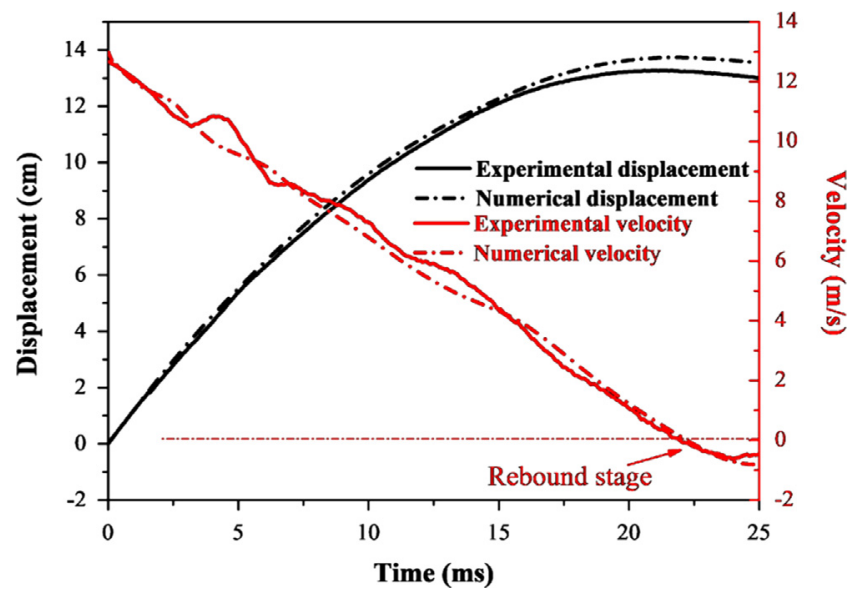

Fig. 6. Typical displacement-time and velocity-time curve at $h=2 \mathrm{~mm}, V=13 \mathrm{~m} / \mathrm{s}$ and $T=300 \mathrm{~K}$.

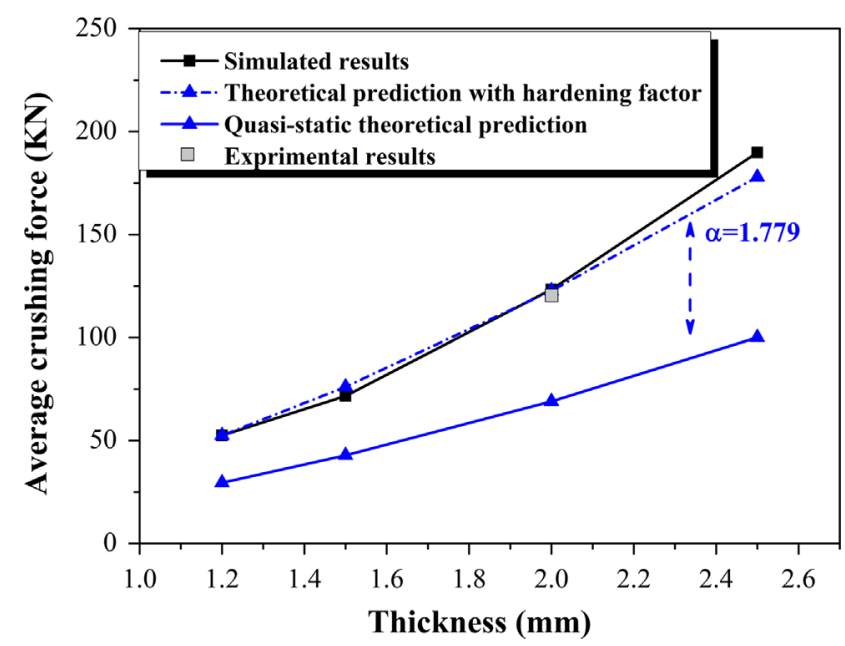

Fig. 7. Numerical results and theoretical prediction of $F_{a v}$ of different thicknesses.

seem less obvious when the impact velocity ranges from $5 \mathrm{~m} / \mathrm{s}$ to $13 \mathrm{~m} / \mathrm{s}$. The average strain rates evaluated by Abramowicz's equation [12] for the diamond buckling mode have only small differences $\left(\dot{\varepsilon}=120 \mathrm{~s}^{-1}, 75 \mathrm{~s}^{-1}\right.$ and $46 \mathrm{~s}^{-1}$ at $V=13 \mathrm{~m} / \mathrm{s}, 8 \mathrm{~m} / \mathrm{s}$ and $5 \mathrm{~m} / \mathrm{s}$, respectively) which will not lead to significant increases in yield strength and plastic flow stress. This result is the reason why the average crushing force does not respond much to the average strain rate. However, stainless steel has strong strain hardening properties before a certain strain (65\% for 304 stainless steel [27]),
Table 3

Average crushing forces at different loading conditions.

\begin{tabular}{llllll}
\hline Conditions $(\boldsymbol{h}=\mathbf{2 . 0} \mathrm{mm})$ & $\begin{array}{l}\text { Impact } \\
\text { energy }(\mathbf{k J})\end{array}$ & $\begin{array}{l}\text { Max. crushing } \\
\text { displacement } \\
(\mathbf{m m})\end{array}$ & $\begin{array}{l}\text { Average } \\
\text { crushing } \\
\text { force }(\mathbf{k N})\end{array}$ & $\boldsymbol{\alpha}$ \\
\hline \multirow{3}{*}{$\mathbf{V}=\mathbf{1 3} \mathbf{~} \mathbf{~} / \mathbf{s}$} & $T=300 \mathrm{~K}$ & 16.9 & 137.11 & 123.32 & 1.779 \\
& $T=523 \mathrm{~K}$ & 16.9 & 178.83 & 94.51 & 1.363 \\
$\mathbf{T}=\mathbf{3 0 0} \mathbf{K}$ & $T=773 \mathrm{~K}$ & 16.9 & 228.91 & 73.82 & 1.065 \\
& $V=8 \mathrm{~m} / \mathrm{s}$ & 6.4 & 53.32 & 120.03 & 1.731 \\
& $V=5 \mathrm{~m} / \mathrm{s}$ & 2.5 & 20.92 & 119.51 & 1.724 \\
\hline
\end{tabular}

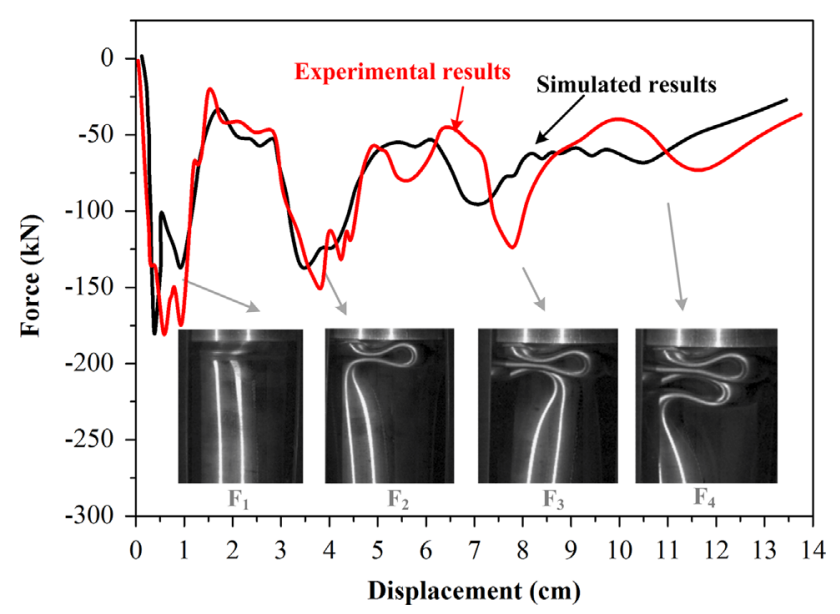

Fig. 8. Transient crushing force-displacement curve at $h=2 \mathrm{~mm}, V=13 \mathrm{~m} / \mathrm{s}$ and $T=300 \mathrm{~K}$.

which contributes greatly to $\alpha$ because large deformations are required to form an intact fold.

\subsection{Transient crushing force}

Fig. 8 shows the typical transient crushing force-displacement curve at $h=2 \mathrm{~mm}, V=13 \mathrm{~m} / \mathrm{s}$ and $T=300 \mathrm{~K}$. The four spikes in the curve represent the actuation of the 1 st, 2 nd and 3rd complete folds and the 4th incomplete fold, respectively. The maximum peak force is $181.83 \mathrm{kN}$ corresponding to the 1 st fold, which is actuated from the dimple at proximal end. The numerical results agree well with the experimental results.

\subsubsection{Local strain rate effect}

A striking feature of the transient crushing force curve is that for the 2nd, 3rd and 4th folds, the corresponding spike values decrease sequentially, which was not observed in most of the static tests. This feature has also appeared in other experimental results [28-31] but has not been deeply studied yet. Generally speaking, for a certain mode of buckling, the spike value of each fold is closely related to the local flow stress of the material, which depends on the local strain, strain rate and temperature during the plastic hinge formation. These effects on materials can be depicted by a $\mathrm{J}-\mathrm{C}$ model, as mentioned in Section 4 . For intact folds, the degree of the deformation is similar, and the induced strain hardening effect is approximately equal. On the contrary, as the velocity of the proximal end continues to decrease as the kinetic energy of the control rod is absorbed by the previous folds, the strain rate of the local material is significantly reduced for the next plastic hinge formation. For a better understanding of this phenomenon, the peak value and corresponding local strain rate for each fold formation are extracted from the numerical results (as shown in Table 4). Although the average strain rate during 
Table 4

Transient velocity and local strain rate during buckling process.

\begin{tabular}{lccc}
\hline Folds & Transient velocity $(\mathbf{m} / \mathbf{s})$ & Local strain rate $\left(\mathbf{s}^{-\mathbf{1}}\right)$ & Peak force $(\mathbf{k N})$ \\
\hline 1st & 12.54 & 410 & 181.83 \\
2nd & 10.50 & 275 & 149.51 \\
3rd & 7.59 & 140 & 102.26 \\
$4 t h$ & 2.15 & 65 & 74.75 \\
\hline
\end{tabular}

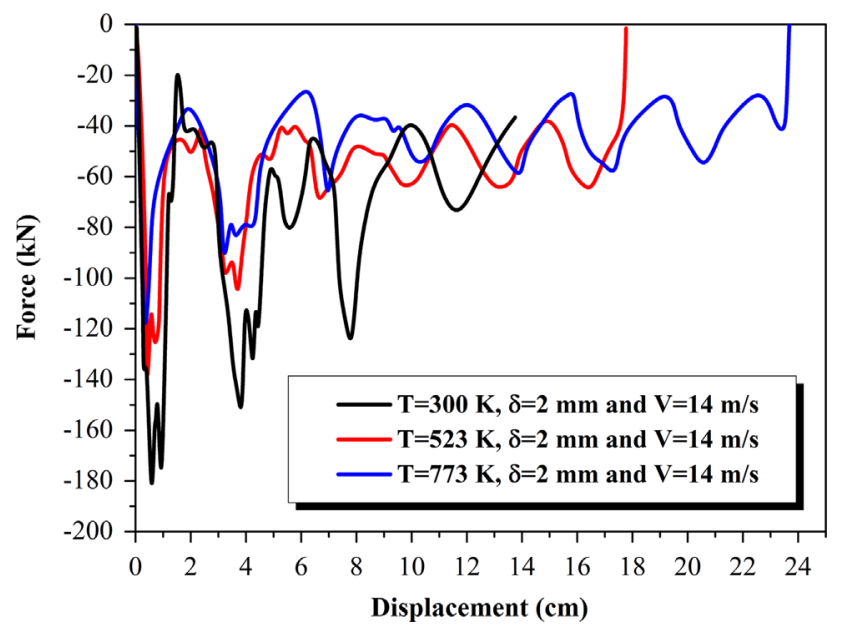

Fig. 9. Typical crushing force-displacement curve at different temperatures.

progressive buckling is relatively low for such an impact velocity $\left(\dot{\varepsilon}=120 \mathrm{~s}^{-1}\right)$, the local strain rate for the plastic hinge formation is quite high and decreases rapidly as the velocity of the proximal end decreases. Thus, this decreasing local strain rate should be taken as characteristic of the decreasing spikes. It could be a common feature in the progressive buckling of thin tubes, which are made of strain-rate sensitive materials when bearing impact loads.

\subsubsection{Temperature effect}

As shown in Fig. 9, the spike values of first three folds decrease significantly as the temperature increases. Additionally, more folds are required at higher temperatures to absorb the impact energy (six folds at $523 \mathrm{~K}$ and seven folds at $773 \mathrm{~K}$ ). In other words, while the crushing force is reduced, the energy absorption capacity per length is also weakened. The effect of temperature on the flow stress of materials agrees well with the numerical models.

\subsubsection{Ratio of maximum and average crushing force}

The ratio of $F_{\max }$ and $F_{a v}$ is defined as $\beta$, which is critical for the security design of absorbers.

$\beta=F_{\max } / F_{a v}$

Generally, the energy absorption capacity is embodied as the cumulative effect of the average crushing force $F_{a v}$ on the displacement. The maximum crushing force $F_{\max }$ is often used as the maximum load for security design. Thus, absorbers with large $\beta$ are less efficient in terms of the energy absorbed per unit length. The local strain rate hardening effect results in a larger $\beta$, as shown in Table 5. As the impact velocity increases, the local strain rate during the 1 st fold formation grows faster than the average strain rate. It is demonstrated that materials are locally strengthened at a higher impact velocity, resulting in a higher $F_{\max }$.

Furthermore, unlike the average strain rate, the local strain rate has a close relationship with the tube thickness. As shown in Table 5 , the local strain rate during the 1 st fold formation increased as the thickness decreased. In the plastic hinge formation process,
Table 5

Variation of $\beta$ at different conditions.

\begin{tabular}{llllrl}
\hline Conditions & & $\begin{array}{l}\text { Local } \dot{\varepsilon} \text { for } \mathbf{1 s t} \\
\text { fold }\left(\mathbf{s}^{-\mathbf{1}}\right)\end{array}$ & $\boldsymbol{F}_{\max }(\mathbf{k N})$ & $\boldsymbol{F}_{\boldsymbol{a v}}(\mathbf{k N})$ & $\boldsymbol{\beta}$ \\
\hline $\boldsymbol{V}=\mathbf{1 3} \mathrm{m} / \mathrm{s}$ & $h=1.2 \mathrm{~mm}$ & 750 & 171.21 & 52.54 & 3.26 \\
$\boldsymbol{T}=\mathbf{3 0 0} \mathbf{K}$ & $h=1.5 \mathrm{~mm}$ & 500 & 173.65 & 71.67 & 2.42 \\
& $h=2.0 \mathrm{~mm}$ & 410 & 181.83 & 123.32 & 1.47 \\
& $h=2.5 \mathrm{~mm}$ & 250 & 198.92 & 189.91 & 1.05 \\
$\boldsymbol{V}=\mathbf{1 3} \mathrm{m} / \mathrm{s}$ & $T=523 \mathrm{~K}$ & 430 & 135.01 & 94.51 & 1.42 \\
$\boldsymbol{h}=\mathbf{2 . 0} \mathbf{~ m m}$ & $T=773 \mathrm{~K}$ & 510 & 115.21 & 73.82 & 1.56 \\
$\boldsymbol{T}=\mathbf{3 0 0} \mathbf{~ K}$ & $V=8 \mathrm{~m} / \mathrm{s}$ & 170 & 174.74 & 120.03 & 1.40 \\
$\boldsymbol{h}=\mathbf{2 . 0 ~} \mathbf{~ m m}$ & $V=5 \mathrm{~m} / \mathrm{s}$ & 95 & 149.98 & 119.51 & 1.19 \\
\hline
\end{tabular}

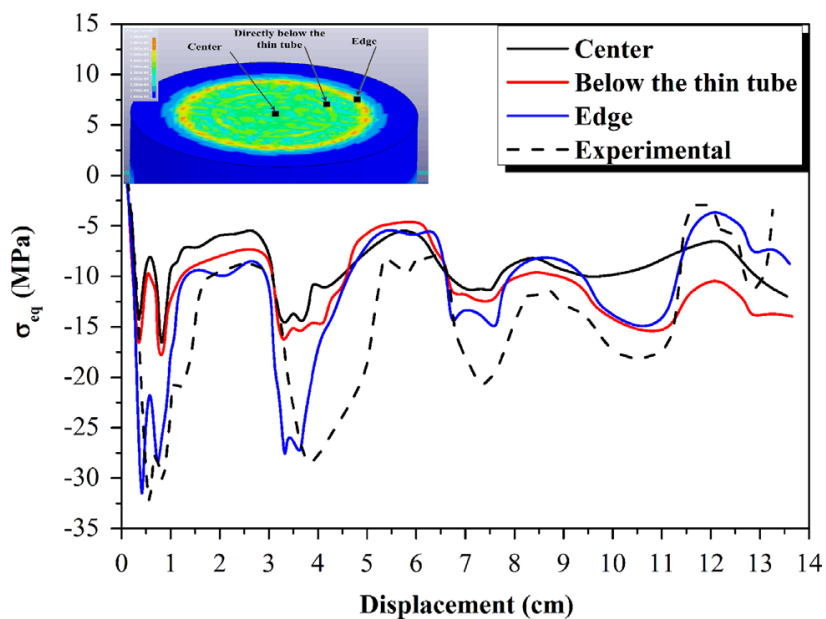

Fig. 10. Stress distribution in the backplate-graphite contact region during the $1 \mathrm{st}$ fold formation at $h=2 \mathrm{~mm}, V=13 \mathrm{~m} / \mathrm{s}$ and $T=300 \mathrm{~K}$.

the thickness reduced the local flexural capacity, and as a result, the local material will deform faster at a certain impact velocity. Thus, the elevated strain rate will strengthen the local material, which maintains the transient crushing force at a certain level. Conversely, the average crushing force is significantly reduced as the tube thickness decreases, which negatively affects the response of $\beta$ to the tube thickness. Thus, the thickness reduction is not an effective method to improve the energy absorbing capacity of the thin tube at an impact condition because the maximum load level does not reduce; however, the crushing displacement increases rapidly.

As the environmental temperature increases, the maximum crushing force exhibits a similar decreasing trend with the average crushing force ( $\beta$ does not significantly change), which is primarily due to the softening effect of the temperature on the plastic flow stress of the material being uniform at any time during the entire buckling process.

\subsection{Stress distribution at the contact surface of graphite}

Graphite, as a type of brittle material with low strength, can be easily destroyed by excessive local pressure. Therefore, it is necessary to prudently assess the stress distribution in the contact region between the backplate and graphite block. For a better understanding of this phenomenon, the typical stress-displacement $(h=2 \mathrm{~mm}, V=13 \mathrm{~m} / \mathrm{s}$ and $T=300 \mathrm{~K}$ ) curve of the three typical regions (center, directly below the thin tube and edge of the interface, respectively) are shown in Fig. 10. The experimental results are obtained from the PVDF sensors located at the edge of the backplate-graphite interface.

The stress in the region directly below the thin tube is slightly greater than that in the central region. In the buckling process, the pressure applied on the upper surface of the backplate is primarily 


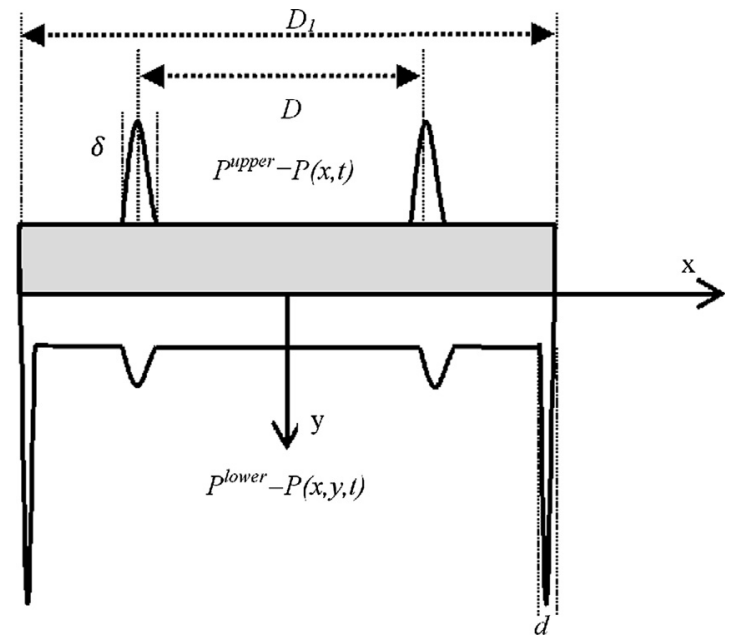

Fig. 11. Schematic diagram of the force analysis of the backplate.

localized in the part that directly contacts the thin tube, as shown in Fig. 11. The upper surface load can be expressed as

$F^{\text {upper }}(t)=2 \pi \int_{(D-h) / 2}^{(D+h) / 2} P^{\text {upper }}(r, t) r d r$

Local deformation of this part will be slightly higher than the surroundings, which induces a higher stress response of the graphite in the corresponding region.

The effect of stress concentration is fairly obvious, i.e., the local stress at the edge during the formation of the 1st fold is much greater than in the other regions. Nevertheless, this phenomenon lessens as the crushing force decreases for the subsequent buckling process, as shown in Fig. 10. The concentration factor $k$ is defined as

$k=\sigma_{\max } / \sigma_{a v}=P_{\max }^{\text {lower }} / P_{a v}^{\text {lower }}$

where $\sigma_{\max }=-P_{\max }^{\text {lower }}$ is the maximum stress derived from the boundary element in the numerical models and $\sigma_{a v}=F_{\max } / s$ is the ratio of the maximum crushing force and backplate area. $k$ is highly related to the local deformation characteristics at the edge, which is represented as a penetration depth of the backplate into the graphite block. In the elastic range of graphite, a deeper penetration will increase the singularity at the edge, such that the stress concentration is exacerbated. Ignoring the uneven stress in the region directly below the thin tube, the pressure distribution can be expressed as

$P^{\text {lower }}= \begin{cases}P(t) & r \leq D_{1}-d \\ k(r, y) P(t) & D_{1}-d \leq r \leq D_{1}\end{cases}$

The parameter $d$ is the width of the stress concentration region. The lower surface load can be expressed as

$$
F^{\text {lower }}(t)=\frac{1}{4} \pi\left(D_{1}-d\right)^{2} P(t)+\frac{1}{2} \pi \int_{D_{1}-d}^{D_{1}} k(r, y) P(t) r d r
$$

If $d \ll D_{1}$, the contribution of the stress concentration region to $F^{\text {lower }}$ is negligible. The lower surface load can be considered most affected by the uniform elastic deformation of the graphite. Thus,

$$
F^{\text {lower }}(t)=\frac{1}{4} \pi D_{1}^{2} P(t)=\frac{y(t)}{4 a} E_{1} \pi D_{1}^{2}
$$

Table 6

Concentration factor at the boundary at different conditions.

\begin{tabular}{llllll}
\hline Conditions & & $\boldsymbol{P}_{\max }(\mathbf{M P a})$ & $\boldsymbol{P}_{\boldsymbol{a v}}(\mathbf{M P a})$ & $\boldsymbol{k}$ & $\boldsymbol{y}_{\max }(\mathbf{m m})$ \\
\hline $\boldsymbol{V}=\mathbf{1 3} \mathbf{~ m} / \mathbf{s}$ & $h=1.2 \mathrm{~mm}$ & 19.09 & 19.07 & 1.00 & 0.07 \\
& $h=1.5 \mathrm{~mm}$ & 22.41 & 19.33 & 1.16 & 0.09 \\
$\boldsymbol{T}=\mathbf{3 0 0} \mathbf{K}$ & $h=2.0 \mathrm{~mm}$ & 33.06 & 20.19 & 1.64 & 0.11 \\
& $h=2.5 \mathrm{~mm}$ & 40.91 & 20.94 & 1.95 & 0.16 \\
$\boldsymbol{V}=\mathbf{1 3} \mathbf{~ m} / \mathbf{s}$ & $T=523 \mathrm{~K}$ & 24.85 & 15.47 & 1.56 & 0.10 \\
$\boldsymbol{h}=\mathbf{2} \mathbf{~ m m}$ & $T=773 \mathrm{~K}$ & 17.95 & 11.18 & 1.44 & 0.07 \\
$\boldsymbol{T}=\mathbf{3 0 0} \mathbf{K}$ & $V=8 \mathrm{~m} / \mathrm{s}$ & 32.23 & 18.39 & 1.72 & 0.12 \\
$\boldsymbol{h}=\mathbf{2} \mathbf{~ m m}$ & $V=5 \mathrm{~m} / \mathrm{s}$ & 28.30 & 15.80 & 1.78 & 0.13 \\
\hline
\end{tabular}

where $a$ is the thickness of the graphite block, $E_{1}$ is Young's modulus. The kinetic equation of the backplate is

$m \frac{\partial^{2} y}{\partial t^{2}}=F^{\text {upper }}(t)-F^{\text {lower }}(t)$

where $m$ is the mass of the backplate. Associating (9) and the initial conditions, $F^{\text {upper }}(t)$ during the 1 st fold formation is described by an exponential decay function $F_{\max } e^{-\gamma t}$

$m \frac{\partial^{2} y}{\partial t^{2}}+\frac{E_{1} \pi D_{1}^{2}}{4 a} y=F_{\max } e^{-\gamma t}, t=0, y=0, \frac{\partial y}{\partial t}=0$

Solving the above equation,

$y=\frac{F_{\max }}{m\left(\gamma^{2}+\theta^{2}\right)}\left(\frac{\gamma}{\theta} \sin \theta t-\cos \theta t+e^{-\gamma t}\right)$

$\theta=D_{1} \sqrt[2]{\frac{E_{1} \pi}{4 a m}}$

The penetration depth is not only related with $F_{\max }$ but also the decaying exponential $\gamma$, which is determined by the time required to form a single fold. As mentioned in Section 5.3, $F_{\max }$ of thinner tubes has a similar value as that of the thicker tubes; however, the time of a single fold formation is shorter. Thus, as the numerical results shows (Table 6), the penetration depth clearly decreased as the thickness decreased, which results in a smaller $k$. At higher temperatures, $F_{\max }$ is significantly weakened by the softening effect on the materials. The stress concentration is released, which results in a smaller penetration depth. For a lower impact velocity, there are two competing effects on the penetration depth, decreasing $F_{\max }$ and increasing fold formation time (smaller $\gamma$ ). The slightly higher $k$ for a lower impact velocity (as shown in Table 6) demonstrates that the latter gains an advantage in the competition.

\section{Conclusions}

In this paper, an energy-absorbing structure based on the progressive buckling phenomena of circular, thin tubes was designed to protect the graphite structure of an HTR from a freefall impact by a massive control rod. Both experimental and numerical methods are performed to study the effect of strain rate and temperature on the average and transient crushing force. Additionally, the stress concentration at the contact surface of the absorber and graphite is prudently evaluated to ensure the structural security of graphite. There is good agreement between the experimental and numerical results, including the buckling modes, crushing force and stress concentration. The following are the main conclusions:

1. The average crushing force in the case of low-velocity impact can be predicted by classical static theories with a correction factor $\alpha$ to represent the effect of loading conditions on material properties. This effect includes the average strain rate 
hardening, strain hardening and temperature softening. The numerical results showed that $\alpha$ should be the material parameter used to describe the effect of the loading conditions on yield strength and is independent of the geometry of the thin tube.

2. In the progressive buckling process, the local strain rate during plastic hinge formation is much higher than the average strain rate and decreases significantly as the transient crushing velocity decreases. As a result, the peak crushing force for each fold formation is sequentially decreased. Furthermore, the local strain rate will increase the ratio of the maximum crushing force and average crushing force $\beta$, which is not the optimal design of an energy-absorbing structure. The maximum crushing force decreases as the impact velocities decrease or the temperature increases but cannot be effectively weakened by reducing the tube thickness.

3. There are stress concentrations at the edge of the backplategraphite contact surface. The stress concentration factor $k$ will worsen as the backplate penetrates further into the graphite block. The penetration depth depends on both the maximum crushing force and the decaying exponential $\gamma$, which is closely related with the time of a single fold formation. The stress concentration will be relieved for a thinner tube or higher temperature but will be slightly exacerbated when bearing a lower impact velocity.

\section{Acknowledgments}

This work is supported by National S\&T Major Project (Grant no. 2008ZX06902-005).

\section{References}

[1] Shirazi SAM, Aghanajafi C, Sadoughi S, Sharifloo N. Design, construction and simulation of a multipurpose system for precision movement of control rods in nuclear reactors. Ann Nucl Energy 2010;37:1659-65.

[2] Takamatsu M, Sekine T, Aoyama T, Uchida M, Kotake S. Demonstration of control rod holding stability of the self actuated shutdown system in Joyo for enhancement of fast reactor inherent safety. J Nucl Sci Technol 2007;44:511-7.

[3] Wang SK, Chen YN, Chyou YP, Yang TT. Identification of damping mechanism of TRR-II reactor control rod during free fall insertion. Nucl Eng Des 2003;226: 243-54.

[4] Yuanqiang W, Xingzhong D, Huizhong Z, Zhiyong H. Design and tests for the HTR-10 control rod system. Nucl Eng Des 2002;218:147-54.

[5] Zhang Z, Wu Z, Wang D, Xu Y, Sun Y, Li F, et al. Current status and technical description of Chinese $2 \times 250$ MWth HTR-PM demonstration plant. Nucl Eng Des 2009;239:1212-9.
[6] Zhang Z, Wu Z, Sun Y, Li F. Design aspects of the Chinese modular hightemperature gas-cooled reactor HTR-PM. Nucl Eng Des 2006;236:485-90.

[7] Sadeghi MM. Design of heavy duty energy absorbers, structural impact and crashworthiness, vol. 2; 1984; 588-604 (16-20 July).

[8] Jones N. Some recent developments and future trends in thin-walled sections for structural crashworthiness. Thin Walled Struct 1998;32:231-3.

[9] Alghamdi AAA. Collapsible impact energy absorbers: an overview. Thin Walled Struct 2001;29:189-213.

[10] Reid SR. Metal tubes as impact energy absorbers. In: Metal forming and impact mechanics. Pergamon Press; 1985; 249-69.

[11] Alexander JM. An approximate analysis of the collapse of thin cylindrical shells under axial loading. Q J Mech Appl Math 1960;13:10-5.

[12] Abramowicz W, Jones N. Dynamic axial crushing of circular tubes. Int J Impact Eng 1984;2:263-81.

[13] Pugsley AG, Macaulay M. The large-scale crumpling of cylindrical columns. QJ Mech Appl Math 1960;13:1-9.

[14] Jones N, De Oliveira JG. Impulsive loading of a cylindrical shell with transverse shear and rotatory inertia. Int J Solids Struct 1983;19:263-79.

[15] Jones N. Recent studies on the dynamic plastic behavior of structures. Appl Mech Rev (United States) 1989;42(4):95-115 (Medium: X; Size).

[16] Karagiozova D, Jones N. Influence of stress waves on the dynamic progressive and dynamic plastic buckling of cylindrical shells. Int J Solids Struct 2001:6723-49.

[17] Karagiozova D, Jones N. Dynamic effects on buckling and energy absorption of cylindrical shells under axial impact. Thin Walled Struct 2001;39:583-610.

[18] Wang Y-C, Huang C-H, Lee Y-C, Tsai H-H. Development of a PVDF sensor array for measurement of the impulsive pressure generated by cavitation bubble collapse. Exp Fluids 2006;41:365-73.

[19] Luo X, Yu S, Sheng X, He S. Temperature effect on IG-11 graphite wear performance. Nucl Eng Des 2005;235:2261-74.

[20] Johnson GR, Cook WH. Fracture characteristics of three metals subjected to various strains, strain rates, temperatures and pressures. Eng Fract Mech 1985;21:31-48.

[21] Wei Y. Experimental research on welding technique and mechanic properties of dissimilar stainless steel laser welding. In: Chinese Academy of Sciences, Institute of Mechnics, Chinese Academy of Sciences, China; 2010.

[22] Greenhalgh E, Meeks C, Clarke A, Thatcher J. The effect of defects on the performance of post-buckled CFRP stringer-stiffened panels. Composites Part A 2003;34:623-33.

[23] Krishnakumar S, Foster CG. Multiple geometric defects: their effect on stability of cylindrical shells. Exp Mech 1991;31:213-9.

[24] Pugsley AG. On the crumpling of thin tubular struts. Q J Mech Appl Math 1979;32:1-7.

[25] Tvergaard V. On the transition from a diamond mode to an axisymmetric mode of collapse in cylindrical shells. Int J Solids Struct 1983;19:845-56.

[26] Wierzbicki T, Optimum design of integrated front panel against crash. Report for Ford Motor Company. Vehicle Component Dept., 15 July 1983.

[27] Wei Y, Experimental research on welding technique and mechanic properties of dissimilar stainless steel laser welding. In: Chinese Academy of Sciences, Institue of Mechanics, China; 2010. p. 158.

[28] Song H-W, Wan Z-M, Xie Z-M, Du X-W. Axial impact behavior and energy absorption efficiency of composite wrapped metal tubes. Int J Impact Eng 2000;24:385-401.

[29] Song H, Fan Z, Tan H, Wang Q. Eccentricity effects on the crushing history and energy absorption of metal tubes collapsed in concertina mode. Tsinghua Sci Technol 2005;10:196-203.

[30] Al Galib D, Limam A. Experimental and numerical investigation of static and dynamic axial crushing of circular aluminum tubes. Thin Walled Struct 2004:42:1103-37.

[31] Jones N. Structural impact. 2nd ed. Cambridge University Press; 2012; 386-7. 